\title{
Optimization of spectral resources allocations using the PAPR criterion in the context of a MPSoC based on RF interconnections
}

\author{
Mohamad HAMIEH ${ }^{1}$, Yves LOUËT ${ }^{2}$, Myriam Ariaudo ${ }^{2}$, and Sebastien QUINTANEL ${ }^{2}$ \\ ${ }^{1}$ Universite de Cergy-Pontoise \\ ${ }^{2}$ Affiliation not available
}

May 5, 2020

\begin{abstract}
This paper presents a new method for the optimization of the allocation of spectral resources. It is based on the variations of the PAPR as a function of the sub-bands allocations for transmission in the context of a MPSoC based on RF interconnections. The method shows that a specific distribution of the frequency sub-bands leads to a diminution of the PAPR which is an important criterion in signal transmission.
\end{abstract}

Keywords:

multiple-access, RF interconnects, PAPR, spectral allocations.

Introduction:

The realization of integrated circuits such as Systems on Chip (SoC), Systems on Package (SoP), or Systems in Package $(\mathrm{SiP})$ is currently a subject of study that raises the development of important research programs. The limitations caused by conventional interconnects are no longer compatible with the new requirements and constraints imposed by emerging technologies in terms of latency, flow rate, and consumption. In this context, many integration technologies have been proposed, like $3 \mathrm{D}^{, 1}$ optical ${ }^{2}$ and/or $\mathrm{RF}$ (radio frequency $)^{3-6}$ interconnect architectures. The 3D interconnects allow the reduction of line lengths but several long interconnects may still exist. Although the optical interconnections allow important flow, they imply a high implementation cost. Concerning RF interconnects, two solutions are proposed: either by radio, ${ }^{7}$ or by a waveguide used as a transmission medium ${ }^{4}$

To implement wired RF interconnect in a Multi-Processor SoC (MPSoC), several central units and memories are grouped in clusters in which classical interconnect (bus) is still used. Each cluster is connected to the $\mathrm{RF}$ interconnect through $\mathrm{RF}$ transceivers $\left(\mathrm{T}_{\mathrm{x}} / \mathrm{R}_{\mathrm{x}}\right)$ including the channel access (figure 1 ).

Figure 1: MPSoC with $32 T_{x} / R_{x}$

Assuming a RF architecture consisting of $32 \mathrm{RF}-\mathrm{NoC}$ interfaces with their own $\mathrm{T}_{\mathrm{x}} / \mathrm{R}_{\mathrm{x}}$ that provide communication between 32 clusters across a transmission line. To allow communications between all clusters simultaneously and in both directions, it takes 992 channels. We propose to use OFDMA that makes reconfigurability easier without changing the architecture of the RF transceivers [1][7][12]. Taking into account the 992 transmissions and the band $\{20-40\} \mathrm{GHz}$ imposed by the technology in the WiNoCoD project (BiCMOS $0.25 \mu \mathrm{m}$ technology), we propose a system of 1024 sub-carriers and a width of $19.531 \mathrm{MHz} /$ subcarrier. The frequency band used is 20 to $40 \mathrm{GHz}$ due to the technology provided to us by NxP Semiconductors for $\mathrm{Tx} / \mathrm{Rx}$ test circuits and the transmission line. 
In this paper we show the different scenarios of possible transmissions between the different clusters of the circuit in terms of subband allocations for each transmission and the consequences in terms of possible bit rates.

The allocation of the subbands depends on the needs of each transmission. The use of OFDMA (figure 2) makes reconfigurability easier without changing the architecture of the RF transceivers since the frequency of the OL is fixed regardless of the subband required for transmission: in our case the total band of Frequency is set by the technology between 20 and $40 \mathrm{GHz}$, and therefore the operating frequency of the OL is set at $30 \mathrm{GHz}$ regardless of the transmission needs.

Figure 2:allocation of spectral resources with OFDMA

There are several criteria for allocating frequency bands such as:

- the proximity / power criterion. In the case of transmission between the transmitter and receiver that are close, the allocated subbands should preferably be those where the attenuation is high. The subbands where the attenuation is minimal, are allocated for transmissions between transmitters and receivers that are distant. In this case, we can optimize the variations of received signals whatever the sources and destinations.

- The variation of the PAPR (Peak to Average Power Ratio) according to the distribution of the subbands can also constitute a criterion. We present an optimization of the allocation of spectral resources by taking the variation of the PAPR as an optimization criterion.

In this paper we study the optimization of the allocation of spectral resources based on the variations of the PAPR as a function of how to allocate the subbands for each transmission.

\section{Definition and problematic}

An OFDM signal is written on a symbol time $\mathrm{T}$ by the following relation:

$$
s[n]=\frac{1}{\sqrt{N}} \sum_{K=0}^{N-1} X_{k} \mathrm{e}^{j 2 \pi K \frac{n}{N}} w[n]
$$

Where: $\mathrm{N}$ is the number of carriers of the OFDM signal, $\mathrm{w}[\mathrm{n}]$ is the rectangular window on the interval $[0$, $\mathrm{N}], \mathrm{X}_{\mathrm{k}}, \mathrm{K}=0 \ldots \mathrm{N}-1$ is the numerical symbol carried by the $\mathrm{k}^{\mathrm{th}}$ carrier at the frequency $\mathrm{f}_{\mathrm{k}}=\mathrm{k} / \mathrm{N}$.

The PAPR represents the ratio between the maximum power of a signal and the average power. The PAPR of $\mathrm{s}[\mathrm{n}]$ is written:

$$
\operatorname{PAPR}\{s[n]\}=\frac{\max _{0 \leq n \leq N}|s[n]|^{2}}{\frac{1}{N} \sum_{n=0}^{N-1}\left\{|s[n]|^{2}\right\}}
$$

PAPR has been recognized as one of the main practical disadvantages of OFDM modulation. OFDM signals have a large amplitude variation leading to a high PAPR. A high PAPR results from the nature of the modulation itself, where several sinusoidal subcarriers will be added to form the signal to be transmitted.

A high PAPR is generally not desirable because it limits the operation of analog components in systems. High PAPR signals require high linearity of the analog circuits, which generally results a higher power consumption and lower efficiency [SEN04] [YVE10].

For example, power amplifiers have maximum efficiency in the saturation zone. On the other hand, it is in this zone that the most important nonlinearities are found. Therefore, the consequences of an amplifier operating in the saturation zone with an OFDM signal of a high PAPR are as follows:

- Degradation of the bit error rate. 
- Rise of secondary lobes in the spectrum.

The PAPR varies with the number of subcarriers. A transmission that requires a large number of subcarriers therefore leads to a high PAPR (figure 3) (Matlab simulation).

Figure 3: Variation of the PAPR as a function of the number of subcarriers.

In the next section we analyze the variations of the PAPR in the baseband as a function of the position of the subbands on the total band or by dividing a band into several subbands (we assume that the sampling must be at least 4 to have a good approximation of the PAPR [TEL01]). This makes it possible to find functional conditions for reducing the PAPR without using reduction methods that can complicate the circuit. Thanks to the OFDMA used for RF interconnections (WiNoCoD), it is possible to allocate several sub-carriers for a transmission. If a certain distribution of the sub-carriers allows a minimum PAPR, then this distribution can be used as a selection criterion for the allocation of spectral resources.

\section{Study of the variation of the PAPR}

With the OFDMA, the assignment of a number of sub-carriers can be done in several ways:

Variation according to the position of the subcarriers

First, we study the variation of the PAPR for 2 portions of $\mathrm{L}$ subcarriers each. The first is fixed and is at the beginning of the total band $\mathrm{B}_{\mathrm{T}}$ and the second is shifted to the right until the end of $\mathrm{B}_{\mathrm{T}}$ as shown in figure 4 .

Figure 4: Synoptic spectrum shift

In a first example, we consider L equal to 32 sub-carriers (625 MHz wide for each spectrum). We perform a simulation by shifting spectrum 2 to $\mathrm{N}=1024$ (in our case the total band is composed of 1024 subcarriers). The result of the simulation shows that the PAPR varies by approximately $0.5 \mathrm{~dB}$ depending on the position of the spectrum 2. The minimum value of the PAPR is obtained for the position of the spectrum 2 at the displacement index 480 (figure5). From this result, we can conclude that if a transmission requires 64 subcarriers, it is preferable to break the band of 64 into 2 subbands of 32 , and to obtain a minimum PAPR the position of the 2 subbands must be the following: spectrum 1 at the beginning of the band and spectrum 2 at the displacement index 480 .

Figure 5: variation of PAPR as a function of the position of the sub-bands.

Position 0 in figure 5 corresponds to the initial position of the simulation where spectra 1 and 2 are at the beginning of the band.

We repeat the simulation for larger spectra in terms of the number of subcarriers by dividing them into 2 sub-spectra of identical widths. The results are shown in Table 1: This table shows the simulation results of the PAPR for a subband at the beginning of the total band and the second moving over the total band of $20 \mathrm{GHz}$.

Table 1: Minimum PAPR according to the position of the spectrum 2

Table 1 shows that each time there is a position of spectrum 2 where the PAPR has a minimum value. Moreover, we notice that the range of variation of the PAPR decreases as the width $\mathrm{L}$ of each spectrum increases while keeping fixed $\mathrm{N}$ at 1024.

Figure 6: Position of spectrum 2 giving a minimum PAPR.

In order to specify the position of the spectrum 2 which gives the minimum value of the PAPR and to define this position as a function of $\mathrm{L}$ and N, we carry out several simulations by varying $\mathrm{L}$ and N. From the simulations, we notice that in all case the minimum value of the PAPR is at the position of the spectrum at $\mathrm{N} /(2-\mathrm{L})$ (figure 6 shows an example for $\mathrm{L}=32$ and $\mathrm{N}=4096$ ). 
This optimization criterion of the PAPR can be used for the choice of the allocation of spectral resources. In the next part we show the variation of the PAPR by dividing a sub-band into several equidistant subbands on the total band BT.

Division of a band into several subbands

In the previous part we saw that in the framework of a division of a band into 2 sub-bands of the same spectral width and for a certain position, we obtain a minimum value of the PAPR. Starting from the same logic, we try to see if the division of a band into several equidistant sub-bands of the same width can reduce the PAPR. To do this, we perform simulations by taking several cases of bandwidth and dividing them into several uniformly distributed subbands equidistantly (Figure 7). The width of each sub-band is 32 sub-carriers (this is the minimum number of sub-carriers allocated for transmission in WiNoCoD).

Figure 7: Division of the Band into several subbands uniformly distributed

Table 2 shows the results of simulations for different cases of division of the band into 2 or more sub-bands.

Table 2: PAPR (dB) resulting from the division into several subbands.

The results obtained in Table 2 show that the decomposition of a band, specified for a given transmission, into several sub-bands uniformly distributed over the total band reduces the PAPR (for example the decomposition of a band of 512 subcarriers in sub-bands of 256, 128, 64 and 32 respectively reduced the PAPR from $8.7 \mathrm{~dB}$ to $8.1 \mathrm{~dB}$ ). So we can conclude that this distribution can be used as a choice for spectral resource allocations. Thanks to this distribution we reduce the PAPR of the transmitted signal without adding reduction methods which complicate the circuit and which increase the energy consumption. This criterion can be complementary to another allocation optimization, for example the allocation of the sub-bands as a function of the distance between the source and the destination.

\section{Conclusion}

We have studied the allocation of spectral resources based on OFDMA modulation which is a multiplexing technique used in telecommunications systems. This technique has several advantages in terms of spectrum sharing and reconfigurability of transmissions while releasing constraints on RF segments that do not need to be reconfigurable. We talked about optimizing the allocation of spectral resources by taking into account as a criterion the variation of the PAPR as a function of the position of the sub-bands. We showed that a specific distribution of the frequency sub-bands leads to a diminution of the PAPR which is an important criterion in signal transmission.

Other criteria could be used such as the proximity / power criterion: the use of bands where attenuation is minimal for long distance transmissions.

\section{REFERENCES}

[1 ] Hamieh M, Quintanel S, Ariaudo M, Louët Y. A new interconnect method for radio frequency intrachip communications using transistors-based distributed access. Microw Opt Technol Lett. 2018;1-6. https://doi.org/10.1002/mop. 31590

[2] ITRS. 2009 edition; Interconnect.

[3] Briére M, Girodias B, Bouchebaba Y, et al.. Design and behavioral modeling tools for optical networkon-chip. In: DATE '04 Proceedings of the Conference on Design, Automation and Test in Europe. Vol 1; 2005:10738.

[4] Chang M-CF, Verbauwhede I, Chien C, et al. Advanced RF/- baseband interconnect schemes for interand intra ULSI Communications. IEEE Trans Electron Dev. 2005;52(7):1271-1285.

[5] Frank Chang M, Cong J, Kaplan A, et al.. CMP network-on-chip overlaid with multi-band RFinterconnect. In: IEEE 14th International Symposium on High Performance Computer Architecture, HPCA; February 16-20, 2008:192-202. 
[6] Socher E, Chang MF. Can RF help CMOS processors? IEEE Commun Mag. 2007;45(8):104-111.

[7] Unlu E, Hamieh M, Moy C, et al. An OFDMA based RF interconnect for massive multi-core processors. In: 2014 Eighth IEEE/ACM International Symposium on Networks-on-Chip (NoCS); September 2014; Ferrara, Italy.

[8] Floyd BA, Hung CM, O KK. Intra-chip wireless interconnect for clock distribution implemented with integrated antennas, receivers and transmitters. IEEE J Solid State Circuits. 2002;37:543551.

[9] Bartek M, Sinaga SM, Burghartz JN. Influence of via-connections on electrical performance of verticallyspaced RF passives. In: Proceedings Electronic Components and Technology. ECTC '05. Vol 2; 2005:15841589 .

[10] Chang M-CF, Roychowdhury VP, Zhang L, Shin H, Qian Y. RF/wireless interconnect for inter- and intra-chip communications. Proc IEEE. 2001;89(4):456-466.

[11] Mombo Boussougou YC, Le Gouguec T, Quere Y, Le Berre D, Martin PM, Huret F. Electromagnetic analysis of RF interconnect. In: 12th IEEE Workshop on Signal Propagation on Interconnects, 2008. SPI 2008:14.

[12] Hamieh M, Ariaudo M, Quintanel S, Louet Y. Sizing of the physical layer of a RF intra-chip communications. In: 2014 21st IEEE International Conference on Electronics, Circuits and Systems (ICECS); 2014:163-166.

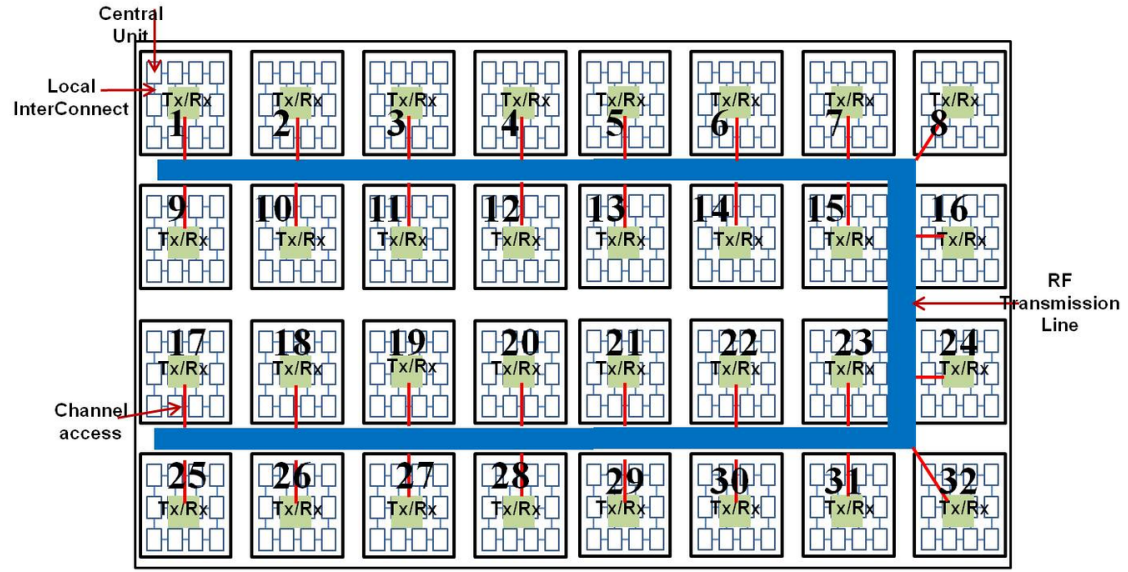

Figure 1: MPSoC with $32 T_{x} R_{x}$

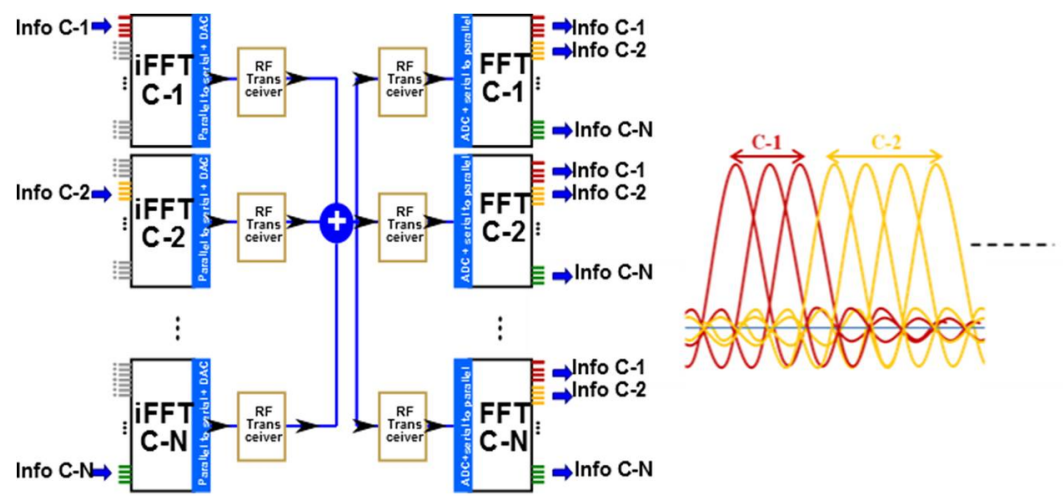

Figure 2: allocation of spectral resources with OFDMA 


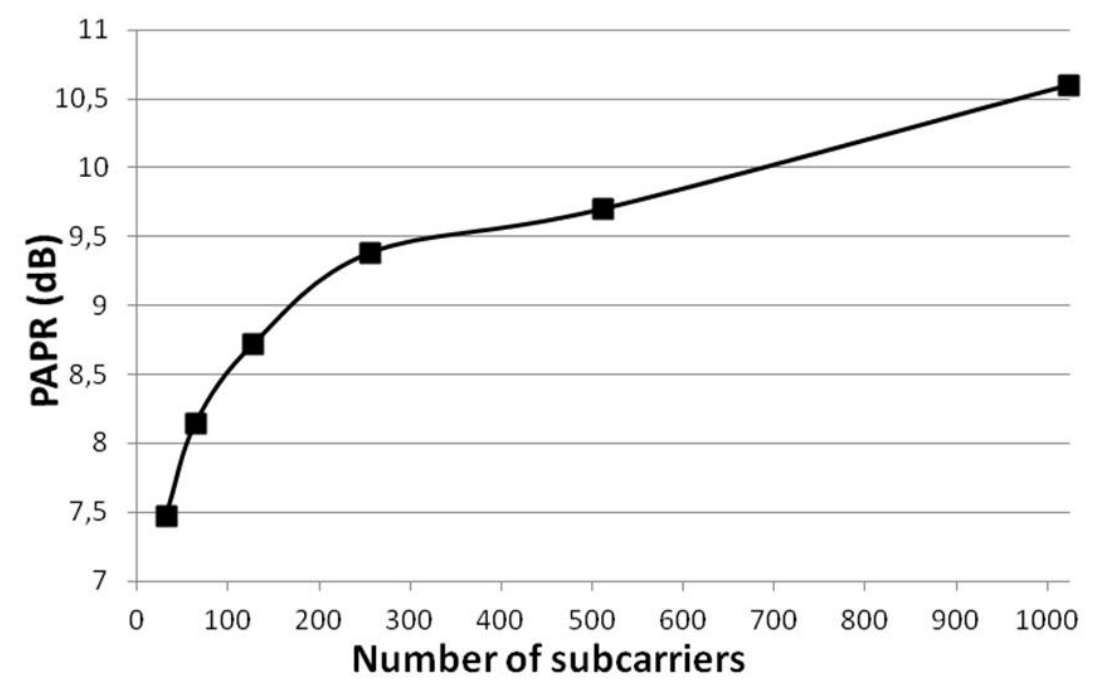

Figure 3: Variation of the PAPR as a function of the number of subcarriers.

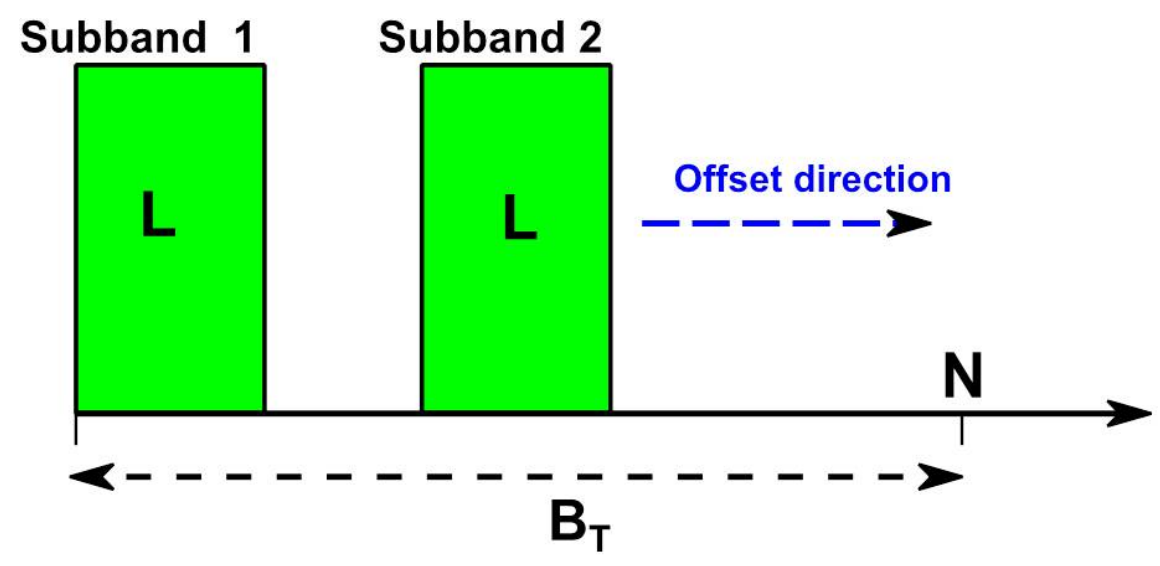

Figure 4: Synoptic spectrum shift 


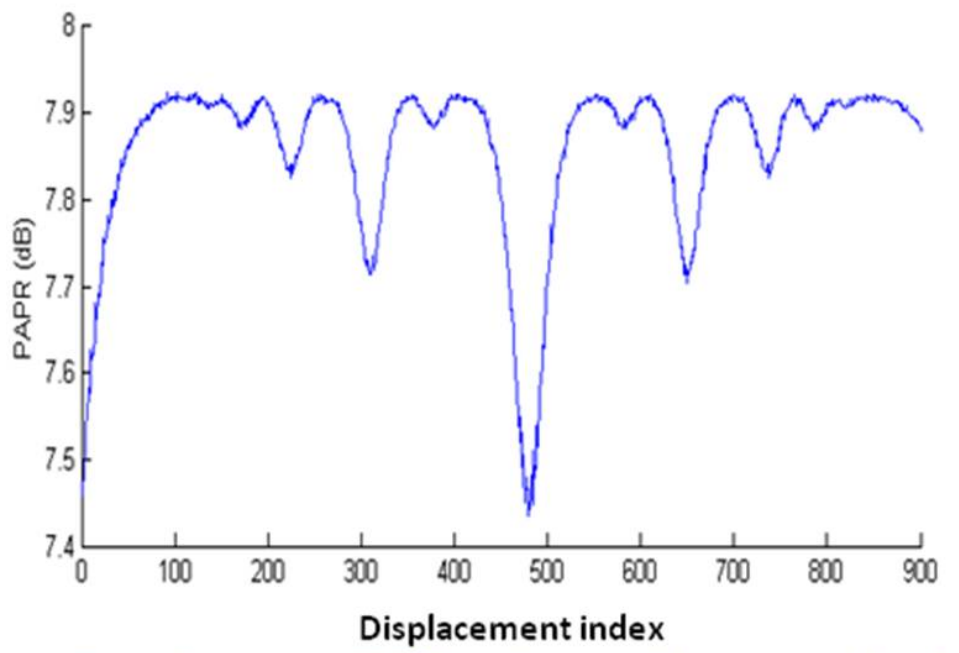

Figure 5: variation of PAPR as a function of the position of the sub-bands.

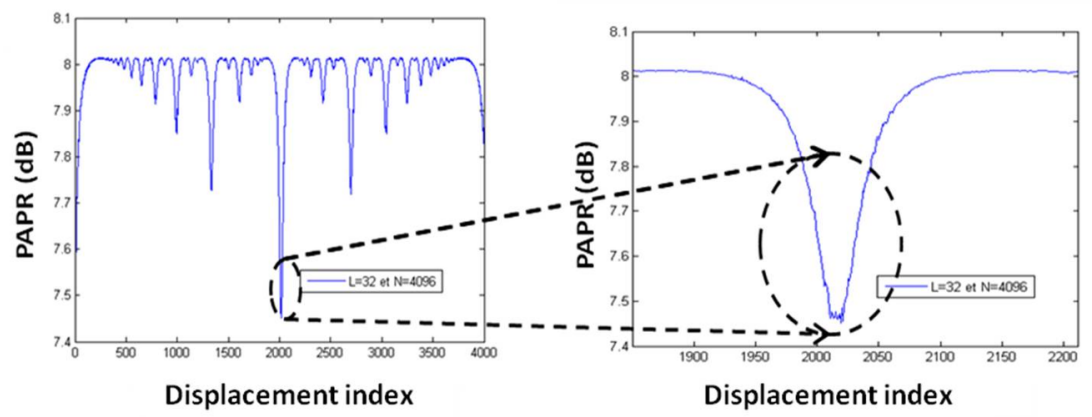

Figure 6: Position of spectrum 2 giving a minimum PAPR.

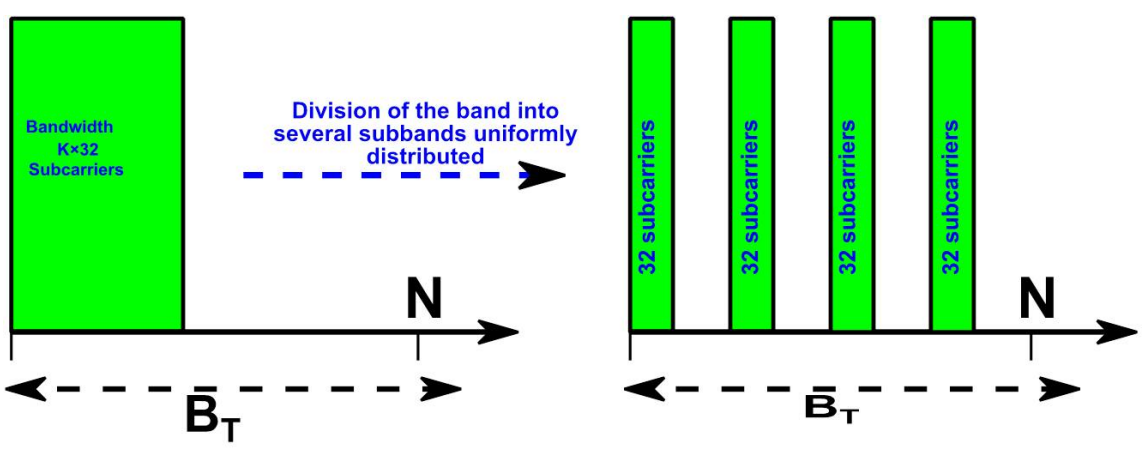

Figure 7: Division of the Band into sever al subbands uniformly distributed 